\title{
Correction: Genome-wide analysis reveals extensive genetic overlap between schizophrenia, bipolar disorder, and intelligence
}

\author{
Olav B Smeland $D^{1}$. Shahram Bahrami ${ }^{1}$. Oleksandr Frei ${ }^{1}$ - Alexey Shadrin ${ }^{1} \cdot$ Kevin O'Connell $^{1}$ - Jeanne Savage $\triangle^{2}$. \\ Kyoko Watanabe $\mathbb{D}^{2}$. Florian Krull ${ }^{1}$. Francesco Bettella ${ }^{1} \cdot$ Nils Eiel Steen $^{1} \cdot$ Torill Ueland $^{1,3}$ - Danielle Posthuma ${ }^{2,4}$. \\ Srdjan Djurovic $\mathbb{D}^{5,6} \cdot$ Anders M Dale ${ }^{7,8,9,10} \cdot$ Ole A Andreassen $\mathbb{I D}^{1}$
}

Published online: 15 July 2019

(c) Springer Nature Limited 2019

\section{Correction to: Molecular Psychiatry}

https://doi.org/10.1038/s41380-018-0332-x

published online 04 January 2019
In the original version of this article, the legend of Fig. 3 inadvertently misstated the GWAS sample size for bipolar disorder as 59,315. The correct sample size is 51,710 . This has been corrected in both the PDF and HTML versions of the article.
Olav B Smeland

o.b.smeland@medisin.uio.no

$\triangle$ Ole A Andreassen

o.a.andreassen@medisin.uio.no

1 NORMENT, KG Jebsen Centre for Psychosis Research, Institute of Clinical Medicine, University of Oslo and Division of Mental Health and Addiction, Oslo University Hospital, 0407 Oslo, Norway

2 Department of Complex Trait Genetics, Center for Neurogenomics and Cognitive Research, Amsterdam Neuroscience, VU University Amsterdam, Amsterdam, The Netherlands

3 Department of Psychology, University of Oslo, Oslo, Norway

4 Department of Clinical Genetics, section Complex Trait Genetics, Neuroscience Campus Amsterdam, VU Medical Center,

Amsterdam, the Netherlands
5 Department of Medical Genetics, Oslo University Hospital, Oslo, Norway

6 NORMENT, KG Jebsen Centre for Psychosis Research, Department of Clinical Science, University of Bergen, Bergen, Norway

7 Department of Cognitive Science, University of California, San Diego, La Jolla, CA 92093, USA

8 Department of Neuroscience, University of California San Diego, La Jolla, CA 92093, USA

9 Department of Radiology, University of California, San Diego, La Jolla, CA 92093, USA

10 Center for Multimodal Imaging and Genetics, University of California San Diego, La Jolla, CA 92093, USA 